\title{
Predação de fêmeas adultas de Podocnemis expansa Schweigger (Testudines, Podocnemididae) por Panthera onca Linnaeus (Carnivora, Felidae), no Estado do Tocantins
}

\author{
Giovanni Salera Júnior ${ }^{1}$, Thiago Costa Gonçalves Portelinha ${ }^{2,4}$ \& Adriana Malvasio ${ }^{3}$ \\ ${ }^{1}$ Instituto Natureza do Tocantins - NATURATINS, \\ Rua Presidente Juscelino Kubitschek, Quadra 25, Lote 11, nº 1353, CEP 77402-100, Gurupi, TO, Brasil \\ ${ }^{2}$ Laboratório de Zoologia de Vertebrados, Departamento de Ciências Biológicas, \\ Escola Superior de Agricultura "Luiz de Queiroz", Universidade de São Paulo - USP, \\ Av. Pádua Dias, 11, CP 09, CEP 13418-900, Piracicaba, SP, Brasil \\ ${ }^{3}$ Laboratório de Ecologia e Zoologia, Universidade Federal do Tocantins - UFT, \\ Av. NS 15, ALCNO, 14, 109 Norte, CP 114, CEP 77001-090, Palmas, TO, Brasil \\ ${ }^{4}$ Autor para correspondência: Thiago Costa Gonçalves Portelinha, e-mail: thiagoportelinha@yahoo.com.br
}

SAlERA-Jr., G., PORTElinhA, T.C.G. \& MALVASIO, A. Predation on adult females of Podocnemis expansa Schweigger (Testudines, Podocnemididae) by Panthera onca Linnaeus (Carnivora, Felidae), in Tocantins State. Biota Neotrop., 9(3): http://www.biotaneotropica.org.br/v9n3/en/abstract?shortcommunication+bn00709032009.

Abstract: Podocnemis expansa (Amazonian giant River turtle) is the largest and more important representative of the Amazonian turtle's fauna because their economic importance as food resource to the indigenous and riverine populations. Aspects related to their reproductive biology, and natural predation of adults in its habitat are poorly known. In order to estimate the predation rates of females of $P$. expansa we have registered the number of turtles with signs of been killed by Panthera onca (jaguar) in a River of the Brazilian Amazon. Jaguar was the only predator of the adult females of P. expansa in the Javaés River beaches (Tocantins State), predating about 3.66\% of the females (33 of 901). Predation always happens at night, when females leave water in order to nest.

Keywords: Amazonian giant River turtle, jaguar, nesting, chelonians, Brazilian Amazon.

SAlERA-Jr., G., PORTElinhA, T.C.G. \& MALVASIO, A. Predação de fêmeas adultas de Podocnemis expansa Schweigger (Testudines, Podocnemididae) por Panthera onca Linnaeus (Carnivora, Felidae), no Estado do Tocantins. Biota Neotrop. 9(3): http://www.biotaneotropica.org.br/v9n3/pt/abstract?shortcommunication+bn00709032009.

Resumo: Podocnemis expansa (tartaruga-da-amazônia) é o maior e mais importante representante da fauna de quelônios da Amazônia devido à sua importância econômica como fonte alimentar para as populações indígenas e ribeirinhas desta região. Apesar disso, os aspectos relacionados à sua biologia reprodutiva, assim como os índices de predação natural de animais adultos em seu habitat são pouco conhecidos. Nessa pesquisa foi averiguado o número de fêmeas de $P$. expansa predadas por Panthera onca (onça-pintada) em um rio da Amazônia Brasileira. A onça-pintada é o único predador das fêmeas adultas de $P$. expansa nas praias do rio Javaés, Estado do Tocantins, predando em média 3,66\% das fêmeas em postura. A predação se dá sempre à noite, quando as fêmeas sobem às praias para nidificar.

Palavras-chave: Tartaruga-da-amazônia, onça-pintada, desova, quelônios, Amazônia Brasileira. 


\section{Introdução}

A diversidade de predadores potenciais dos quelônios é grande, estendendo-se de insetos, que podem predar os ovos (Vogt 1981, Maros et al. 2003), a uma grande variedade de vertebrados. Alguns relatos indicam que as taxas de sobrevivência até a idade adulta de muitas espécies de quelônios ficam entre 1 e $2 \%$ (Ferri 2002).

A grande maioria dos trabalhos sobre a predação natural de quelônios enfatiza a perda de ninhos e filhotes, pois esse tipo de predação é a mais recorrente, mas há de se considerar também que os adultos (machos e fêmeas) podem ser predados por diferentes animais, que utilizam variadas estratégias de captura (Pritchard 1979, Pritchard \& Trebbau 1984). Os crocodilianos (crocodilos e jacarés) incluem em sua dieta uma grande variedade de quelônios aquáticos (Ross 1989). Algumas aves têm desenvolvido táticas especiais para capturar e predar quelônios terrestres, quebrando os cascos arremessando-os das alturas em rochedos (Ferri 2002). Jabutis que vivem em matas e florestas podem ser predados por grandes carnívoros (onças), que fazem aberturas no casco desses animais e se alimentam da carne e vísceras (Emmons 1989).

Podocnemis expansa (tartaruga-da-amazônia) é o maior quelônio de água doce da América do Sul, chegando a medir até $107 \mathrm{~cm}$ de comprimento da carapaça e podendo pesar $60 \mathrm{~kg}$. Esta espécie nidifica nas praias em grupos de centenas e até milhares de fêmeas, preferencialmente após a meia-noite (Pritchard \& Trebbau 1984, Ernst \& Barbour 1989, Dupre et al. 2007). As fêmeas caminham lentamente pela praia, escavam um ninho profundo e depositam em média 100 ovos, retornando para o rio após o fechamento do ninho. Todas essas etapas desde a saída para a postura e retorno ao rio podem levar mais de 2 horas, e é durante a nidificação que as fêmeas estão mais vulneráveis, permitindo a aproximação de predadores (Vanzolini 1967, Alho \& Pádua 1982, Pritchard \& Trebbau 1984).

Na Amazônia brasileira existem poucos estudos relatando os índices de predação natural de quelônios adultos (Goeldi 1906, Jerozolimsky 2005). Nesse trabalho averiguou-se o número de fêmeas de Podocnemis expansa (tartaruga-da-amazônia) predadas por Panthera onca (onça-pintada) entre os anos de 2000 e 2004 em praias do Rio Javaés, Estado do Tocantins.

\section{Material e Métodos}

Os dados dessa pesquisa foram coletados em cinco praias (Canguçu, Comprida, Coco, Goiaba e Bonita) da margem direita do rio Javaés, no entorno do Parque Nacional do Araguaia e da Área de Proteção Ambiental Bananal/ Cantão (oeste do Estado do Tocantins) durante cinco estações reprodutivas (2000-2004) (Figura 1).

Essas praias foram monitoradas nos períodos da manhã e tarde, durante os principais meses de desova de $P$. expansa nesta região (setembro e outubro) para a localização das fêmeas predadas e para a identificação dos ninhos, através dos rastros deixados pela fêmea durante a postura, conforme Malvasio et al. (2002) e Salera Júnior (2005). O monitoramento foi realizado diariamente para reduzir a possibilidade de não localizarmos algum ninho onde, eventualmente, as pegadas poderiam ter sido recobertas por areia.

Quando da identificação de uma fêmea predada, os dados relativos à predação (e.g. data, praia, predador, período da predação em relação à desova, consumo da carcaça e local onde a carcaça foi abandonada) eram anotados em ficha de campo e os cascos (carapaça e plastrão) eram mensurados e pesados seguindo Molina (1989, 1995). A identificação do predador a partir dos rastros e pegadas baseou-se em Becker \& Dalponte (1999).

\section{Resultados e Discussão}

Para a correta relação do percentual de fêmeas predadas, adotouse o número total de posturas (ninhos) mais o número de fêmeas predadas antes de terem realizado a desova. Portanto, durante as cinco estações reprodutivas (2000-2004), foram encontrados 873 ninhos e 28 fêmeas predadas antes de realizarem a nidificação, o que corresponde a um total de 901 fêmeas em postura. O número total de ninhos encontrados pode ser interpretado como a quantidade real de desovas para cada ano, tendo em vista que a metodologia empregada para localização dos mesmos é confiável e utilizada pela maioria dos projetos de conservação de quelônios, como é o caso do "Projeto Quelônios da Amazônia”" (IBAMA 1989).

Panthera onca é o único predador das fêmeas adultas de P. expansa nas praias do rio Javaés. Verificou-se que 33 fêmeas (limites de 2-13) foram predadas durante as cinco estações de desova, o que representa uma média de 3,66\% fêmeas predadas em postura (limites 0,88-7,30\%). A predação dá-se sempre à noite, quando as fêmeas de $P$. expansa sobem às praias para desovar.

Dentre as cinco praias monitoradas, só foi registrada a predação de fêmeas nas praias Canguçu $(84,85 \%)$, Coco $(9,09 \%)$ e Bonita $(6,06 \%)$, que são as que recebem anualmente mais de $80 \%$ das desovas. Observou-se também uma variação anual no número de fêmeas predadas nestas praias (Tabela 1). Possivelmente, estas variações podem estar associadas à quantidade de fêmeas que estão nidificando na praia ou por alterações no comportamento alimentar de $P$. onca e pela disponibilidade de outras presas na natureza.

No monitoramento diário das praias foram verificados que vários exemplares de $P$. onca, individualmente, em pares ou em grupo visitam freqüentemente tais locais. Cabe ressaltar que a visita em grupo caracterizava-se pela presença de um indivíduo adulto acompanhado por um ou dois filhotes.

Um dos mais antigos registros da predação de quelônios amazônicos por onças é apresentado por Humboldt (1852), informando que durante o período reprodutivo, inúmeras fêmeas de $P$. expansa são predadas por $P$. onca, sendo viradas e o seu conteúdo retirado do casco sem danificação da carapaça. Outro registro antigo é apresentado por Goeldi (1906) que através de informações coletadas entre indígenas da região amazônica brasileira, afirma que jabutis (Chelonoidis spp.) são comumente predados por onças.

Emmons (1989) em coleta de dados realizada na Estação Biológica de Cocha Cashu, Parque Nacional Manu (Peru) e na região

Tabela 1. Número de fêmeas adultas de $P$. expansa predadas por Panthera onca para cada estação de desova, em diferentes praias do rio Javaés, Tocantins, Brasil.

Table 1. Location of the adult females of $P$. expansa predated by Panthera onca for each nesting season in different beaches of the Javaés River, Tocantins State, Brazil.

\begin{tabular}{clc}
\hline Ano & Praias & Fêmeas predadas \\
\hline 2000 & Canguçu & 11 \\
2001 & Canguçu & 1 \\
& Bonita & 1 \\
2002 & Canguçu & 9 \\
& Coco & 2 \\
2003 & Bonita & 2 \\
2004 & Canguçu & 5 \\
Total & Canguçu & 2 \\
\hline
\end{tabular}




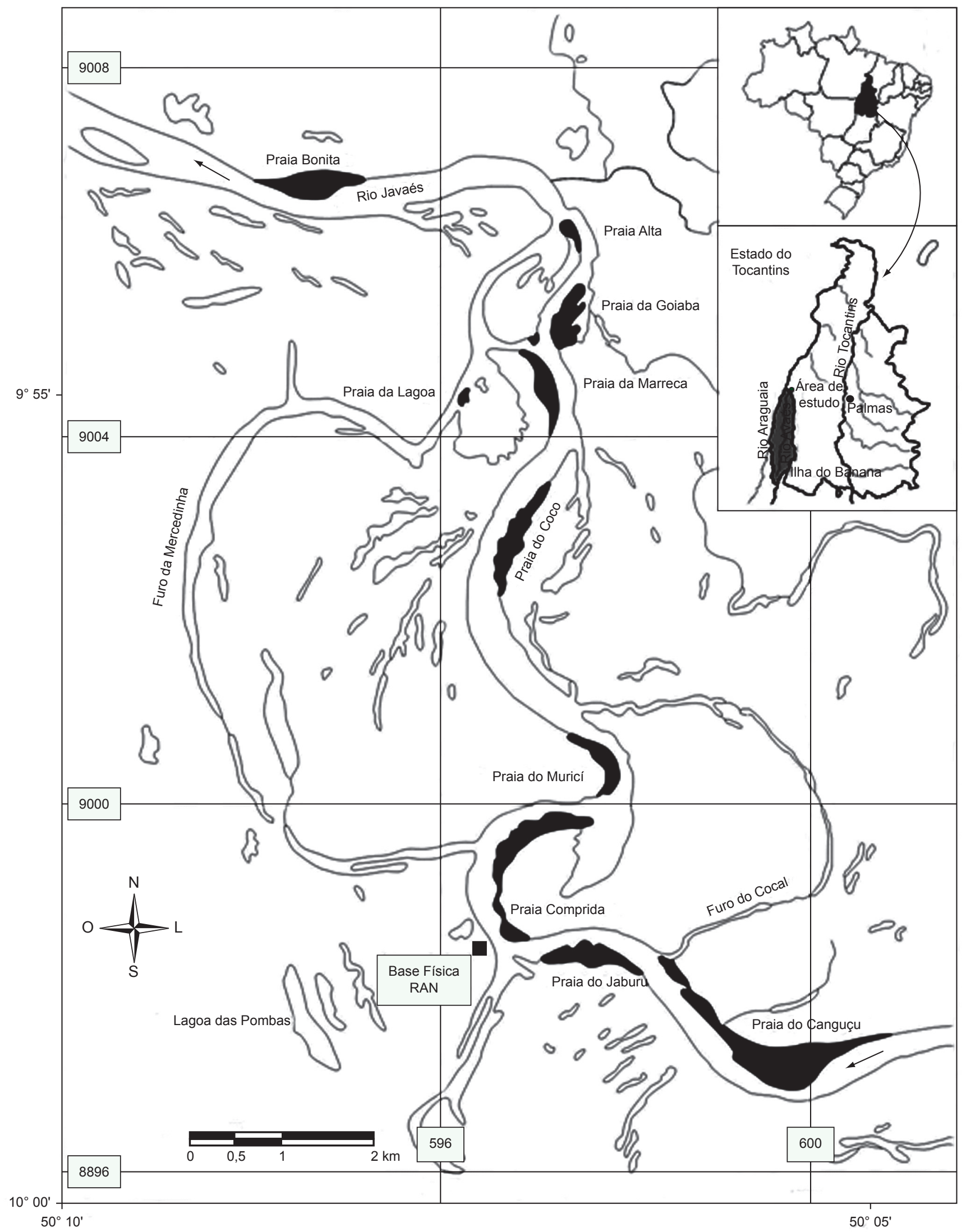

Figura 1. Localização da área de estudo no entorno do Parque Nacional do Araguaia, Estado do Tocantins.

Figure 1. Study area around the Araguaia National Park, Tocantins State. 


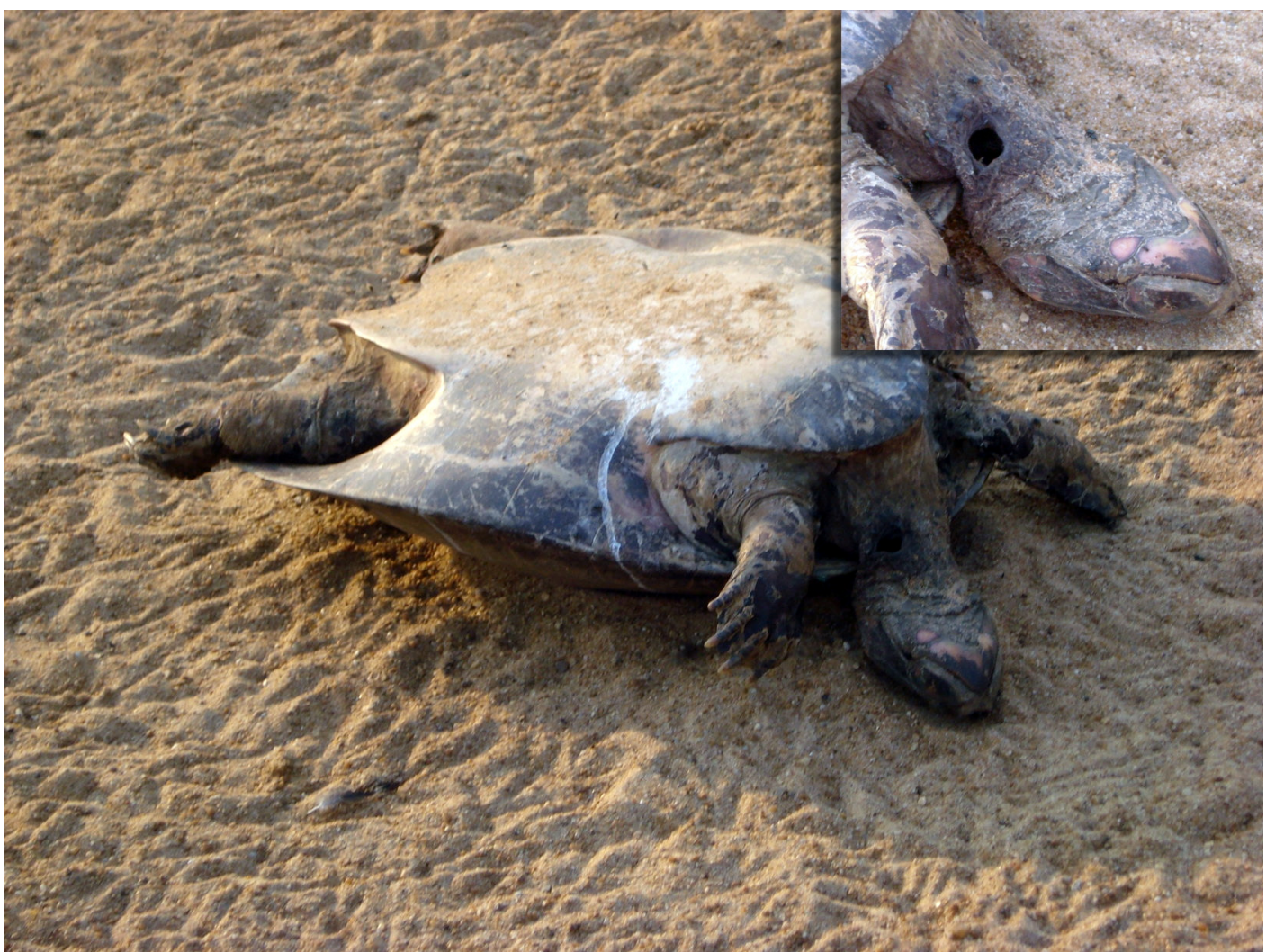

Figura 2. Fêmea adulta de $P$. expansa predada por $P$. onca após realizar desova na praia Canguçu, no detalhe marcas do ataque no pescoço da fêmea.

Figure 2. Adult female of $P$. expansa predated by $P$. onca after the nesting time in the Canguçu beach, details show the marks of the attack on the female neck.

do rio Arataye (Guiana Francesa) apresentou dados da predação de exemplares de Chelonoidis denticulata (jabuti), P. unifilis (tracajá) e Platemys platycephala (cágado) por $P$. onca. A autora descreve que esses quelônios têm o casco quebrado, geralmente na porção superior da carapaça, por onde o animal retira e consome seu conteúdo (carne e vísceras). Além da abertura deixada no casco, verificam-se também inúmeras ranhuras e perfurações deixadas pelas garras e dentes do predador.

Os cascos das fêmeas de $P$. expansa predadas apresentaram um comprimento médio da carapaça de $72,1 \mathrm{~cm}(\mathrm{n}=14$, limites de 66-79,2 cm) e massa média do casco de 7,3 $\mathrm{kg}(\mathrm{n}=4$, limites de $6,7-7,8 \mathrm{~kg}$ ). Jerozolimski (2005) registrou a predação de um macho adulto de $C$. denticulata, com comprimento linear da carapaça igual a 27,6 cm, que estava sendo monitorado por radiotelemetria à cerca de um ano na Terra Indígena Kayapó (Estado do Pará) por $P$. onca, exatamente da forma descrita por Emmons (1989). O autor registrou ainda em outros inúmeros jabutis a presença de cicatrizes no casco, indicando ataques de onças.

No rio Javaés, todas as fêmeas predadas apresentavam-se viradas sob suas carapaças, na posição de decúbito dorsal. Os rastros e as marcas deixadas na areia da praia possibilitavam a localização da carcaça do animal, assim como a identificação do predador. Essas marcas indicavam que as fêmeas foram arrastadas viradas (com a carapaça para baixo), sendo puxadas pela cabeça, pescoço ou patas. Verificou-se somente o consumo das patas e do conteúdo interno (carne e vísceras), não sendo registrado o consumo de partes do pescoço e cabeça.

Panthera onca preda alguns grandes mamíferos, freqüentemente através de mordidas no crânio (Schaller \& Vasconcelos 1978).
Observou-se que quando da predação das fêmeas, a cabeça e o pescoço encontravam-se bem projetados para fora do casco e continham marcas de mordidas (Figura 2) e o casco podia, muitas vezes, apresentar marcas de ranhuras, e às vezes, verificava-se a quebra de partes dos escudos marginais da carapaça e/ ou anais e gulares do plastrão, diferentemente do registrado por Humboldt (1852), que informa não haver dano às carapaças quando das predações.

Facure \& Giaretta (1996) estudando diversos carnívoros na Reserva Florestal da Companhia Vale do Rio Doce, município de Linhares (Estado do Espírito Santo), determinaram a dieta de $P$. onca através da análise de fezes coletadas e identificaram oito itens alimentares, dentre os quais, duas espécies de quelônios, $C$. denticulata e Acanthochelys radiolata (cágado). Garla et al. (2001) corroboram esse estudo registrando para a mesma localidade, que $C$. denticulata está entre as seis espécies de presas encontradas com maior frequencia em fezes de $P$. onca.

Taber et al. (1997) informam que o jabuti Chelonoidis chilensis faz parte da dieta de $P$. onca no Paraguai. Rabinowitz (1986) também observou a presença de quelônios aquáticos na dieta de $P$. onca, em Belize, na América Central. Apesar de registrarem quelônios na dieta de $P$. onca nessas diferentes localidades, os autores não apresentam dados relacionados à predação, em parte, por terem trabalhado apenas com a análise de fezes e por terem encontrado apenas um pequeno número de carcaças de quelônios.

As praias de estudo estão inseridas em área de influência de duas unidades de conservação (Parque Nacional do Araguaia e APA Bananal/ Cantão) e contam com a fiscalização do IBAMA, que possui uma base no local, o que justifica a ausência de predação humana sobre as populações de quelônios, de forma que $P$. onca pode 
ser considerada o único predador das fêmeas em postura naquela localidade.

Através das informações biométricas das fêmeas predadas, seria possível gerar um indicativo da quantidade de ovos perdidos que poderiam estar eclodindo nas praias do rio Javaés, através da relação existente entre o tamanho da fêmea e o número de ovos depositados pela espécie estudada (Valenzuela 2001, Bonach et al. 2006). Essas informações são de grande relevância para projetos de conservação e os conhecimentos sobre as taxas de predação dessas fêmeas em postura poderiam subsidiar uma melhor compreensão da estrutura dessas populações. Deve-se salientar, ainda, a importância de estudos com este enfoque a fim de averiguar os possíveis fatores que estariam influenciando na variação dos ataques por estes predadores ao longo do tempo nesta região e em outros locais com características similares.

\section{Agradecimentos}

Este trabalho só foi possível com o apoio recebido pelo NATURATINS, através de Isac Bráz da Cunha. O apoio às etapas de campo foi dado pelo IBAMA, RAN/ IBAMA, Instituto Ecológica, Earthwatch Institute e UFT. Gostaríamos de agradecer a Divaldo Rezende, Stephano Merlim, Mariluce Messias e Maria Tereza do Instituto Ecológica, Antônia Lúcia Mendes do Carmo e Selma Cristina do IBAMA e Yeda de Lucena Bataus do RAN/ ICMBio. Agradecemos também aos voluntários do Earthwatch Institute que participaram ativamente da coleta de dados em 2001, 2002 e 2003.

\section{Referências Bibliográficas}

ALHO, C.J.R. \& PÁDUA, L.F.M. 1982. Reproductive parameters and nesting behavior of the Amazon turtle Podocnemis expansa (Testudinata: Pelomedusidae) in Brazil. Can. J. Zool. 60(1):97-103.

BECKER, M. \& DALPONTE, J.C. 1999. Rastros de mamíferos silvestres brasileiros: um guia de campo. 2 ed. UnB/IBAMA, Brasília, 180 p.

BONACH, K., PIÑA, C.I. \& VERDADE, L.M. 2006. Allometry of reproduction of Podocnemis expansa in Southern Amazon Basin. Amphibia-Reptilia. 27:55-61.

DUPRE, A., DEVAUX, B. \& BONIN, F. 2007. Turtles of the World. A \& C Black Publishers Ltd, London, $416 \mathrm{p}$.

EMMONS, L.H. 1989. Jaguar Predation on Chelonians. J. Herpetol. 23(3):311-314.

ERNST, C.H. \& BARBOUR, R.W. 1989. Turtles of the World. Smithsonian Institution Press, Washington, $328 \mathrm{p}$.

FACURE, K.G. \& GIARETTA, A.A. 1996. Food habits of carnivores in a coastal Atlantic Forest of southeastern Brazil. Mammalia. 60(3):499-502.

FERRI, V. 2002. Turtles \& Tortoises: a firefly guide. Firefly Books, Ontario, $256 \mathrm{p}$.

GARLA, R.C., SETZ, E.Z.F. \& GOBBI, N. 2001. Jaguar (Panthera onca) food habits in Atlantic Rain Forest of Southeastern Brazil. Biotropica. 33(4):691-696.

GOELDI, E.A. 1906. Chelonios do Brazil. Bol. Mus. Para. Emílio Goeldi. 4(1-4):699-756.

HUMBOLDT, A. 1852. A personal narrative of travels to the equinoctal regions of America during 1799-1804. H.G. Bohn, London, 521 p. (v. 2).
Instituto Brasileiro do Meio Ambiente e dos Recursos Naturais Renováveis - IBAMA. 1989. Projeto Quelônios da Amazônia: 10 anos. IBAMA, Brasília, $119 \mathrm{p}$.

JEROZOLIMSKI, A. 2005. Ecologia de populações dos jabutis Geochelone denticulata e G. carbonaria (Cryptodira: Testudinidae) no território da aldeia A'Ukre, Terra Indígena Kaiapó, sul do Pará. Tese de Doutorado, Universidade de São Paulo, São Paulo, 264 p.

MALVASIO, A., SOUZA, A.M., FERREIRA Jr., P.D., REIS, E.S. \& SAMPAIO, F.A.A. 2002. Temperatura de incubação dos ovos e granulometria dos sedimentos das covas relacionadas à determinação sexual em Podocnemis expansa (Schweigger, 1812) e P. unifilis (Troschel, 1848) (Testudines, Pelomedusidae). Publ. Avulsas Inst. Pau Bras. de Hist. Nat. 5(1):11-25.

MAROS, A., LOUVEAUX, A., GODFREY, M.H. \& GIRONDOT, M. 2003. Scapteriscus didactylus (Orthoptera, Gryllotalpidae), predator of leatherback turtle eggs in French Guiana. Mar. Ecol. Prog. Ser. 249:289-296.

MOLINA, F.B. 1989. Observações sobre a biologia e o comportamento de Phrynops geoffranus (Schweigger, 1812) em cativeiro (Reptilia, Testudines, Chelidae). Dissertação de Mestrado, Universidade de São Paulo, São Paulo, 185 p.

MOLINA, F.B. 1995. Observações sobre a biologia e o comportamento reprodutivo de Trachemys dorbignyi (Duméril \& Bibron, 1835) em cativeiro (Reptilia, Testudines, Emydidae). Tese de Doutorado, Universidade de São Paulo, São Paulo, 307 p.

PRITCHARD, P.C.H. 1979. Encyclopedia of Turtles. T.F.H. Publ. Inc., Neptune, $895 \mathrm{p}$.

PRITCHARD, P.C.H. \& TREBBAU, P. 1984. The Turtles of Venezuela. Society for the Study of Amphibians and Reptiles, Ohio, $414 \mathrm{p}$.

RABINOWITZ, A.R \& NOTTINGHAM JR, B.G. 1986. Ecology and behaviour of the Jaguar (Panthera onca) in Belize, Central America. J. Zool. 210(11):149-159.

ROSS, C.A 1989. Crocodiles and alligators. Facts on File, New York, 240 p.

SALERA Jr., G. 2005. Avaliação da biologia reprodutiva, predação natural e importância social em quelônios com ocorrência na bacia do Araguaia. Dissertação de Mestrado, Universidade Federal do Tocantins, Palmas, $202 \mathrm{p}$.

SCHALLER, G.B. \& VASCONCELOS, J.M.C. 1978. Jaguar predation on capybara. Z. Saugetierk. 43:296-301.

TABER, A.B., NOVARO, A.J., NERIS, N. \& COLMAN, F.H. 1997 The food habits of sympatric jaguar and puma in the Paraguayan Chaco. Biotropica. 29(2):204-213.

VALENZUELA, N. 2001. Maternal effects on life-history traits in the Amazonian giant river turtle Podocnemis expansa. J. Herpetol. 35(3):368-378.

VANZOLINI, P.E. 1967. Notes on the nesting behavior of Podocnemis expansa in the Amazon Valley (Testudines, Pelomedusidae). Pap. Avulsos Zool. 20(17):191-215.

VOGT, R.C. 1981. Turtle egg (Graptemys: Emydidae) infestation by fly larvae. Copeia. 2:457 459.

Recebido em 06/02/09 Versão reformulada recebida em 28/05/09 Publicado em 01/07/09 